\title{
Sharper Existence and Uniqueness Results for Solutions to Third-Order Boundary Value Problems
}

\author{
Saleh S. Almuthaybiri ${ }^{a, b}$ and Christopher C. Tisdell ${ }^{a}$ \\ ${ }^{a}$ School of Mathematics and Statistics, The University of New South Wales \\ UNSW, 2052 Sydney, Australia \\ ${ }^{b}$ Department of Mathematics, College of Sciences and Arts, Qassim University \\ Qassim, Oqlatu's Soqoor, Saudi Arabia \\ E-mail(corresp.): cct@unsw.edu.au \\ E-mail: s.almuthaybiri@qu.edu.sa
}

Received August 15, 2019; revised April 14, 2020; accepted April 16, 2020

\begin{abstract}
The purpose of this note is to sharpen Smirnov's recent work on existence and uniqueness of solutions to third-order ordinary differential equations that are subjected to two- and three-point boundary conditions. The advancement is achieved in the following ways. Firstly, we provide sharp and sharpened estimates for integrals regarding various Green's functions. Secondly, we apply these sharper estimates to problems in conjunction with Banach's fixed point theorem. Thirdly, we apply Rus's contraction mapping theorem in a metric space, where two metrics are employed. Our new results improve those of Smirnov by showing that a larger class of boundary value problems admit a unique solution.
\end{abstract}

Keywords: third-order boundary value problem, existence and uniqueness of solutions, Rus's contraction mapping theorem, two metrics, three-point boundary conditions, sharp estimates.

AMS Subject Classification: 34B15.

\section{Introduction}

Recently, Smirnov [7] considered the following third-order ordinary differential equation

$$
x^{\prime \prime \prime}+f(t, x)=0, \quad t \in[a, b] .
$$

Above, Smirnov makes the assumptions $f:[a, b] \times \mathbb{R} \rightarrow \mathbb{R}$ with $f(t, 0) \neq 0$ for all $t \in[a, b]$ so that the trivial solution $x \equiv 0$ is excluded. Smirnov [7] subjected

This is an Open Access article distributed under the terms of the Creative Commons Attribution License (http://creativecommons.org/licenses/by/4.0/), which permits unrestricted use, distribution, and reproduction in any medium, provided the original author and source are credited. 
(1.1) to the following three-point boundary conditions:

$$
x(a)=0, \quad x^{\prime}(a)=0, \quad x(b)=k x(\eta),
$$

where $a<\eta<b$ and $k \in \mathbb{R}$. Observe that if $k=0$ then (1.2) collectively becomes two-point conditions.

Smirnov [7] skillfully developed a theory regarding existence and uniqueness of solutions for the boundary value problem (BVP) of the form (1.1)-(1.2) via use of Banach's contraction mapping [3] in a complete metric space and also established interesting properties of the associated Green's function. By a solution we mean a three-times continuously differentiable function (that is, $\left.x \in C^{3}([a, b])\right)$ that satisfies the BVP $(1.1)-(1.2)$.

The purpose of the present note is to sharpen Smirnov's existence and uniqueness results for the BVP (1.1)-(1.2). This is achieved in three directions and in complementary ways. Firstly, we provide sharp and sharpened estimates for integrals regarding various Green's functions. Secondly, these sharper estimates are applied to problems via Banach's [3] fixed point theorem. Thirdly, we apply Rus's contraction mapping theorem [6] in a metric space, where two metrics are employed. Our new results improve those of Smirnov by illustrating that a larger class of these kinds of problems admit a unique solution.

The following result is due to Banach [3] and forms a well-known and powerful theorem concerning fixed points of operators. It will form a key element in our strategy of extending Smirnov's work.

Theorem 1 [Banach, [3]]. Let $X$ be a nonempty set and let $d$ be a metric on $X$ such that $(X, d)$ forms a complete metric space. If the mapping $T: X \rightarrow X$ satisfies

$$
d(T x, T y) \leq \alpha d(x, y), \text { for some } 0<\alpha<1 \text { and all } x, y \in X
$$

then there is a unique $z \in X$ such that $T z=z$.

Although Banach's theorem has been widely applied by researchers over the past 100 years, we will see that our sharp and sharpened estimates on the Green's functions can be combined with Banach's theorem to advance the current state of knowledge regarding the existence and uniqueness of solutions to our BVP (1.1)-(1.2). Thus, we take the position that by continuing to look at classical and long-standing ideas and problems from alternative points of view we can develop and advance the state of current knowledge.

The following result due to Rus [6] will also form a core part of our approach to extending Smirnov's work.

Theorem 2 [Rus, [6]]. Let $X$ be a nonempty set and let $d$ and $\delta$ be two metrics on $X$ such that $(X, d)$ forms a complete metric space. If the mapping $T: X \rightarrow X$ is continuous with respect to $d$ on $X$ and:

$$
\begin{array}{ll}
d(T x, T y) \leq c \delta(x, y), & \text { for some } c>0 \text { and all } x, y \in X \\
\delta(T x, T y) \leq \alpha \delta(x, y), & \text { for some } 0<\alpha<1 \text { and all } x, y \in X
\end{array}
$$

then there is a unique $z \in X$ such that $T z=z$. 
When compared with more well known techniques from fixed point theory, such as Theorem 1, there has been limited research involving applications of Theorem 2 to explore the questions of existence and uniqueness of solutions to ordinary differential equations. It is surprising that Theorem 2 has not received more attention from researchers, given that it was published more than 40 years ago. Perhaps its sheltered state has more to do with the human tendency to favour approaches that are more well known. That is, humankind tends to continue to tread along methodological paths that are more well travelled without exploring alternative perspectives and techniques. However, developing alternative perspectives are important as they can open up new ways of thinking and working $[8,9]$.

Theorem 2 differs from Theorem 1. For example, Theorem 2 involves two metrics which may not necessarily be equivalent. In addition, the underlying space in Theorem 2 is assumed to be complete with respect to the first of these metrics, but not necessarily complete with respect to the second metric. The operator is assumed to be contractive with respect to the second metric. As we will discover, it is these very properties that have the potential to advance recent (or longer-standing) results on existence and uniqueness of solutions to BVPs. Thus, we take the position that Theorem 2 forms an important, underappreciated and untapped tool that has the potential to open up new lines of inquiry and thus is most worthy of attention. For more recent applications of fixed point theory with two metrics, see [2].

Consider the set of real-valued functions that are defined and continuous on $[a, b]$ and denote this space by $X:=C([a, b])$. For functions $x, y \in C([a, b])$ consider the following two metrics on our $X$ :

$$
\begin{aligned}
d(x, y) & :=\max _{t \in[a, b]}|x(t)-y(t)| \\
\delta(x, y) & :=\left(\int_{a}^{b}|x(t)-y(t)|^{p} d t\right)^{1 / p}, \quad p>1 .
\end{aligned}
$$

For $d$ in $(1.5)$, the pair $(C([a, b]), d)$ forms a complete metric space. For $\delta$ in (1.6), the pair $(C([a, b]), \delta)$ forms a metric space, but it is not complete. A useful relationship between the two metrics on our $X$ that we will draw on is

$$
\delta(x, y) \leq(b-a)^{1 / p} d(x, y), \quad \text { for all } x, y \in C([a, b]) .
$$

This work is organized as follows. In Section 2 we establish sharp and sharper estimates on the integrals regarding various Green's functions. In Section 3 these sharper estimates are applied to problems via the fixed point theorems of Banach and Rus to ensure existence and uniqueness of solutions; and we also establish some results regarding the convergence and error of approximations. We also devote discussion to fully illustrate the nature of the advancements made via use of remarks and examples in Section 4.

\section{Sharper and sharpest estimates}

In this section we establish improved inequalities for integrals involving various Green's function that are associated with the BVP (1.1)-(1.2). 
The BVP (1.1)-(1.2) can be recast as an equivalent integral equation [7, pp.173-174]

$$
x(t)=\int_{a}^{b} G(t, s) f(s, x(s)) d s, \quad t \in[a, b]
$$

where

$$
G(t, s):=R(t, s)+\frac{k(t-a)^{2}}{(b-a)^{2}-k(\eta-a)^{2}} R(\eta, s)
$$

and $R$ is given explicitly by

$$
R(t, s)=\frac{1}{2} \begin{cases}(t-a)^{2}(b-s)^{2} /(b-a)^{2}-(t-s)^{2}, & \text { for } a \leq s \leq t \leq b \\ (t-a)^{2}(b-s)^{2} /(b-a)^{2}, & \text { for } a \leq t \leq s \leq b\end{cases}
$$

The following result establishes the non-negativity of the function $R$ and will be useful in developing our estimates on the integrals of $R$ and $|G|$.

Theorem 3. The function $R(t, s)$ in (2.3) satisfies $R \geq 0$ on $[a, b] \times[a, b]$.

Proof. From (2.3) we can see that the case showing $R(t, s) \geq 0$ on the region $a \leq t \leq s \leq b$ is obvious due the the squared form of the function therein.

The remaining situation to show $R(t, s) \geq 0$ on the region $a \leq s \leq t \leq b$ involves some algebraic manipulation in the following manner. From (2.3), if we apply the formula for the difference of two squares, then we see that for $a \leq s \leq t \leq b$ we have

$$
\begin{aligned}
& \frac{(t-a)^{2}(b-s)^{2}}{2(b-a)^{2}}-\frac{(t-s)^{2}}{2} \\
= & \frac{1}{2}\left[\frac{(t-a)(b-s)}{b-a}+(t-s)\right]\left[\frac{(t-a)(b-s)}{b-a}-(t-s)\right] \geq 0 .
\end{aligned}
$$

The non-negativity follows from the fact that each expression contained in the square brackets of the above product is non-negative and so the product in question is non-negative. For example, in the first square bracket we have a sum of two non-negative terms; while in the second square bracket we may equivalently write the terms in as $(s-a)(b-t) /(b-a) \geq 0$.

Let us utilize Theorem 3 to form the following sharp and sharper estimates on the integrals of various Green's functions for the BVP (1.1)-(1.2). The estimates will be of a helpful form for our analysis in Section 3. The estimates are also of independent interest.

Theorem 4. The function $R(t, s)$ in (2.3) satisfies

$$
\int_{a}^{b} R(t, s) d s \leq \frac{2}{81}(b-a)^{3}, \quad \text { for all } t \in[a, b] .
$$

Inequality (2.4) is sharp in the sense that it is the best inequality possible. 
Proof. For all $t \in[a, b]$ we have

$$
\begin{aligned}
& \int_{a}^{b} R(t, s) d s=\int_{a}^{t} R(t, s) d s+\int_{t}^{b} R(t, s) d s \\
= & \int_{a}^{t} \frac{(t-a)^{2}(b-s)^{2}}{2(b-a)^{2}}-\frac{(t-s)^{2}}{2} d s+\int_{t}^{b} \frac{(t-a)^{2}(b-s)^{2}}{2(b-a)^{2}} d s \\
= & \frac{(t-a)^{2}}{6(b-a)^{2}}\left[-(b-t)^{3}+(b-a)^{3}\right]-\frac{(t-a)^{3}}{6}+\frac{(t-a)^{2}(b-t)^{3}}{6(b-a)^{2}} \\
= & \frac{(t-a)^{2}(b-t)}{6} .
\end{aligned}
$$

Now, if we define $r(t):=\int_{a}^{b} R(t, s) d s$, then an application of basic calculus reveals that

$$
\max _{t \in[a, b]} r(t)=\max _{t \in[a, b]} \frac{(t-a)^{2}(b-t)}{6}=\frac{2}{81}(b-a)^{3} .
$$

In particular, the maximum of $r$ on $[a, b]$ is attained when $t=a+\frac{2}{3}(b-a)$ and this illustrates that the inequality (2.4) is sharp.

Remark 1. Smirnov [7, p.175] forms the estimate

$$
\int_{a}^{b}|R(t, s)| d s \leq \frac{(b-a)^{3}}{3}, \quad \text { for all } t \in[a, b] .
$$

If we compare (2.5) with our sharper estimate (2.4) then it is easy to see that Theorem 4 extends [7, Proposition 3.].

An analogue of Theorem 4 for $G$ now follows.

Theorem 5. The function $G(t, s)$ in (2.2) satisfies

$$
\int_{a}^{b}|G(t, s)| d s \leq(b-a)^{3}\left[\frac{2}{81}+\frac{|k|(b-a)^{2}}{3\left|(b-a)^{2}-k(\eta-a)^{2}\right|}\right], \text { for all } t \in[a, b],
$$

where we have assumed $k(\eta-a)^{2} \neq(b-a)^{2}$ with $a<\eta<b$.

Proof. Similarly to the proof of Theorem 4, for $t \in[a, b]$, we have

$$
\begin{aligned}
& \int_{a}^{b}|G(t, s)| d s=\int_{a}^{b}\left|R(t, s)+\frac{k(t-a)^{2}}{(b-a)^{2}-k(\eta-a)^{2}} R(\eta, s)\right| d s \\
\leq & \int_{a}^{b}|R(t, s)|+\left|\frac{k(t-a)^{2}}{(b-a)^{2}-k(\eta-a)^{2}}\right||R(\eta, s)| d s \\
= & \frac{1}{6}(t-a)^{2}(b-t)+\frac{|k|(t-a)^{2}}{\left|(b-a)^{2}-k(\eta-a)^{2}\right|} \frac{1}{6}\left[(\eta-a)^{2}(b-\eta)+(\eta-t)^{3}\right] \\
\leq & \frac{2}{81}(b-a)^{3}+\frac{|k|(b-a)^{2}}{\left|(b-a)^{2}-k(\eta-a)^{2}\right|} \frac{1}{3}(b-a)^{3} \\
= & (b-a)^{3}\left[\frac{2}{81}+\frac{|k|(b-a)^{2}}{3\left|(b-a)^{2}-k(\eta-a)^{2}\right|}\right] .
\end{aligned}
$$


Above, we employed the fact that $R \geq 0$ and (2.4). Thus we have established $(2.6)$.

Remark 2. Smirnov [7, p.176] forms the estimate

$$
\int_{a}^{b}|G(t, s)| d s \leq \frac{(b-a)^{3}}{3}\left[1+\frac{|k|(b-a)^{2}}{\left|(b-a)^{2}-k(\eta-a)^{2}\right|}\right], \text { for all } t \in[a, b] .
$$

If we compare (2.7) with our sharp estimate (2.6) then it is easy to see that Theorem 5 extends [7, Proposition 4.].

\section{$3 \quad$ Existence and uniqueness}

In this section we establish novel results for the existence and uniqueness of solutions via applications of fixed point approaches.

\subsection{Banach fixed point approach with sharp inequalities}

Let us now apply the results of Section 2 to the existence and uniqueness of solutions to the BVP (1.1)-(1.2) via Banach's fixed point theorem.

Theorem 6. Let $f:[a, b] \times \mathbb{R} \rightarrow \mathbb{R}$ be continuous, let $f(t, 0) \neq 0$ for all $t \in[a, b]$ and let $L$ be a non-negative constant such that

$$
|f(t, u)-f(t, v)| \leq L|u-v|, \quad \text { for all }(t, u),(t, v) \in[a, b] \times \mathbb{R} .
$$

If $k(\eta-a)^{2} \neq(b-a)^{2}$ with $a<\eta<b$ and

$$
L(b-a)^{3}\left[\frac{2}{81}+\frac{|k|(b-a)^{2}}{3\left|(b-a)^{2}-k(\eta-a)^{2}\right|}\right]<1,
$$

then the BVP (1.1)-(1.2) has a unique (nontrivial) solution in $C^{3}([a, b])$.

Proof. Consider the operator $T: C([a, b]) \rightarrow C([a, b])$ defined by

$$
(T x)(t):=\int_{a}^{b} G(t, s) f(s, x(s)) d s, \quad t \in[a, b] .
$$

In view of (2.1) we wish to show that there exists a unique $x \in C([a, b])$ such that $T x=x$. Every such solution will also lie in $C^{3}([a, b])$ as can be directly shown by differentiating (2.1) and confirming the continuity.

To establish the existence and uniqueness to $T x=x$, we show that the conditions of Theorem 1 hold. Consider the pair $(X, d)=(C([a, b]), d)$ which forms a complete metric space. For $x, y \in C([a, b])$ and $t \in[a, b]$, consider

$$
\begin{aligned}
& |(T x)(t)-(T y)(t)| \\
& \leq \int_{a}^{b}|G(t, s)| \mid f(s, x(s))-f\left(s, y(s)\left|d s \leq \int_{a}^{b}\right| G(t, s)|L| x(s)-y(s) \mid d s\right. \\
& \leq L d(x, y) \int_{a}^{b}|G(t, s)| d s \leq L(b-a)^{3}\left[\frac{2}{81}+\frac{|k|(b-a)^{2}}{3\left|(b-a)^{2}-k(\eta-a)^{2}\right|}\right] d(x, y),
\end{aligned}
$$


where we have applied (2.6).

Taking the maximum of both sides of the inequality (3.2) over $[a, b]$ we thus have for all $x, y \in C([a, b])$

$$
d(T x, T y) \leq L(b-a)^{3}\left[\frac{2}{81}+\frac{|k|(b-a)^{2}}{3\left|(b-a)^{2}-k(\eta-a)^{2}\right|}\right] d(x, y),
$$

and in light of (3.1) we see that $T$ satisfies all of the conditions of Theorem 1. Thus, the operator $T$ has a unique fixed point in $C([a, b])$. This solution is also in $C^{3}([a, b])$ and we have equivalently shown that the BVP (1.1)-(1.2) has a unique (nontrivial) solution.

Remark 3. Smirnov's result [7, Theorem 1] assumes

$$
L \frac{(b-a)^{3}}{3}\left[1+\frac{|k|(b-a)^{2}}{\left|k(\eta-a)^{2}-(b-a)^{2}\right|}\right]<1 .
$$

If we compare (3.3) with our (3.1) then we can see that (3.1) forms a less restrictive condition.

The following results are a consequence of Theorem 1 holding for the operator $T$ therein, see [10, Theorem 1.A]. We will use it to form the following results that involve approximations to the unique solution $x$ of the BVP (1.1)-(1.2).

Remark 4. Let the conditions of Theorem 6 hold. If we recursively define a sequence of approximations $x_{n}=x_{n}(t)$ on $[a, b]$ via

$$
x_{0}:=0, \quad x_{n+1}(t):=\int_{a}^{b} G(t, s) f\left(s, x_{n}(s)\right) d s, \quad n=1,2, \ldots
$$

then:

- the sequence $x_{n}$ converges to the solution $x$ of (1.1)-(1.2) with respect to the $d$ metric and the rate of convergence is given by

$$
d\left(x_{n+1}, x\right) \leq L(b-a)^{3}\left[\frac{2}{81}+\frac{|k|(b-a)^{2}}{3\left|(b-a)^{2}-k(\eta-a)^{2}\right|}\right] d\left(x_{n}, x\right) ;
$$

- for each $n$, an a priori estimate on the error is

$$
d\left(x_{n}, x\right) \leq \frac{\left(L(b-a)^{3}\left[\frac{2}{81}+\frac{|k|(b-a)^{2}}{3\left|(b-a)^{2}-k(\eta-a)^{2}\right|}\right]\right)^{n}}{1-L(b-a)^{3}\left[\frac{2}{81}+\frac{|k|(b-a)^{2}}{3\left|(b-a)^{2}-k(\eta-a)^{2}\right|}\right]} d\left(x_{1}, 0\right)
$$

- for each $n$, an a posteriori estimate on the error is

$$
d\left(x_{n+1}, x\right) \leq \frac{L(b-a)^{3}\left[\frac{2}{81}+\frac{|k|(b-a)^{2}}{3\left|(b-a)^{2}-k(\eta-a)^{2}\right|}\right]}{1-L(b-a)^{3}\left[\frac{2}{81}+\frac{|k|(b-a)^{2}}{3\left|(b-a)^{2}-k(\eta-a)^{2}\right|}\right]} d\left(x_{n+1}, x_{n}\right) .
$$




\subsection{Fixed point approach with two metrics}

Let us now state and prove our results on existence and uniqueness of solutions to (1.1)-(1.2) where we employ two metrics under Rus's theorem.

Theorem 7. Let $f:[a, b] \times \mathbb{R} \rightarrow \mathbb{R}$ be continuous, let $f(t, 0) \neq 0$ for all $t \in[a, b]$ and let $L$ be a non-negative constant such that

$$
|f(t, u)-f(t, v)| \leq L|u-v|, \quad \text { for all }(t, u),(t, v) \in[a, b] \times \mathbb{R} .
$$

If $k(\eta-a)^{2} \neq(b-a)^{2}$ with $a<\eta<b$ and there are constants $p>1$ and $q>1$ such that $1 / p+1 / q=1$ with

$$
L\left(\int_{a}^{b}\left(\int_{a}^{b}|G(t, s)|^{q} d s\right)^{p / q} d t\right)^{1 / p}<1,
$$

then the BVP (1.1)-(1.2) has a unique (nontrivial) solution in $C^{3}([a, b])$.

Proof. Consider the operator $T: C([a, b]) \rightarrow C([a, b])$ defined by

$$
(T x)(t):=\int_{a}^{b} G(t, s) f(s, x(s)) d s, \quad t \in[a, b] .
$$

In light of (2.1) we want to show that there exists a unique $x \in C([a, b])$ such that $T x=x$. Such a solution will also lie in $C^{3}([a, b])$ as can be directly shown by differentiating (2.1) and confirming the continuity.

To establish the existence and uniqueness to $T x=x$, we show that the conditions of Theorem 2 hold. Consider the pair $(X, d)=(C([a, b]), d)$ which forms a complete metric space. In addition, consider the metric $\delta$ on $X$ where $p>1$. For $x, y \in C([a, b])$ and $t \in[a, b]$, consider

$$
\begin{aligned}
& |(T x)(t)-(T y)(t)| \leq \int_{a}^{b}|G(t, s)| \mid f(s, x(s))-f(s, y(s) \mid d s \\
& \leq \int_{a}^{b}|G(t, s)| L|x(s)-y(s)| d s \leq\left(\int_{a}^{b}|G(t, s)|^{q} d s\right)^{1 / q} \\
& \times L\left(\int_{a}^{b}|x(s)-y(s)|^{p} d s\right)^{1 / p} \leq L \max _{t \in[a, b]}\left(\int_{a}^{b}|G(t, s)|^{q} d s\right)^{1 / q} \delta(x, y) .
\end{aligned}
$$

Above, we have used (3.4) and Hölder's inequality [4,5] to obtain (3.6). Thus, defining

$$
c:=L \max _{t \in[a, b]}\left(\int_{a}^{b}|G(t, s)|^{q} d s\right)^{1 / q}
$$

we see that

$$
d(T x, T y) \leq c \delta(x, y), \quad \text { for some } c>0 \text { and all } x, y \in C([a, b])
$$

and so the inequality (1.3) of Theorem 2 holds. 
Now, for all $x, y \in C([a, b])$ we may apply (1.7) to (3.7) to obtain

$$
d(T x, T y) \leq c \delta(x, y) \leq c(b-a)^{1 / p} d(x, y)
$$

Thus, given any $\varepsilon>0$ we can choose $\Delta=\varepsilon / c(b-a)^{1 / p}$ so that $d(T x, T y)<\varepsilon$ whenever $d(x, y)<\Delta$. Hence $T$ is continuous on $C([a, b])$ with respect to the $d$ metric.

Finally, we show that $T$ is contractive on $C([a, b])$ with respect to the $\delta$ metric, that is, the inequality (1.4) in Theorem 2 holds. From (3.6), for each $x, y \in C([a, b])$ consider

$$
\left(\int_{a}^{b}|(T x)(t)-(T y)(t)|^{p} d t\right)^{1 / p} \leq L\left(\int_{a}^{b}\left(\int_{a}^{b}|G(t, s)|^{q} d s\right)^{p / q} d t\right)^{1 / p} \delta(x, y),
$$

and so we obtain

$$
\delta(T x, T y) \leq L\left(\int_{a}^{b}\left(\int_{a}^{b}|G(t, s)|^{q} d s\right)^{p / q} d t\right)^{1 / p} \delta(x, y) .
$$

From our assumption (3.5), we thus have

$$
\delta(T x, T y) \leq \alpha \delta(x, y)
$$

for some $\alpha<1$ and all $x, y \in C([a, b])$.

Thus, Theorem 2 is applicable and the operator $T$ has a unique fixed point in $C([a, b])$. This solution is also in $C^{3}([a, b])$ and we have equivalently shown that the BVP (1.1)-(1.2) has a unique (nontrivial) solution.

For the choices $p=2$ and $q=2$ our Theorem 7 becomes the following new result.

Theorem 8. Let $f:[a, b] \times \mathbb{R} \rightarrow \mathbb{R}$ be continuous, let $f(t, 0) \neq 0$ for all $t \in[a, b]$ and let $L$ be a non-negative constant such that

$$
|f(t, u)-f(t, v)| \leq L|u-v|, \quad \text { for all }(t, u),(t, v) \in[a, b] \times \mathbb{R} .
$$

If $k(\eta-a)^{2} \neq(b-a)^{2}$ with $a<\eta<b$ and

$$
L\left(\int_{a}^{b}\left(\int_{a}^{b}|G(t, s)|^{2} d s\right) d t\right)^{1 / 2}<1
$$

then the BVP (1.1)- (1.2) has a unique (nontrivial) solution in $C^{3}([a, b])$.

Remark 5. The left hand side of the condition (3.8) does not appear to be particularly pleasant to calculate by hand. Indeed, even when we employed Maple to evaluate the left hand side of (3.8) we produced a very complicated expression that took up nearly an entire page (even after attempts at "simplification"). Thus, we have elected not to expressly include this bound for general intervals $[a, b]$ but we have the Maple code [1] for those who are interested. However, as we will see below, we can discuss some special cases that shed some light on the situation. 


\section{Examples, comparisons and remarks}

Let us discuss the nature of the advancement of our theorems through exemplification, comparisons and remarks.

Remark 6. In the case $[a, b]=[0,1]$, Smirnov's result $[7$, Theorem 1] for (1.1), (1.2) assumes (3.4) holds for some constant $L$ such that

$$
\frac{1}{3} L\left[1+|k| /\left|1-k \eta^{2}\right|\right]<1
$$

Observe the limit on the size of the Lipschitz constant $L$ governed by (4.1). Given $k$ and $\eta$, for sufficiently small $L$, the inequality (4.1) will hold.

Let us illustrate how condition (4.1) is sharpened through our Theorem 8 by discussing an example. Consider $k=1, \eta=1 / 2$. In this situation, Smirnov's condition (4.1) becomes

$$
L<9 / 7 \text {. }
$$

Whereas the left hand side of (3.8) can be evaluated (for example, using Maple [1]) with our particular values of $k, a, b$ and $\eta$, which leads to

$$
\int_{0}^{1} G(t, s)^{2} d s=\frac{4}{27} t^{7}-\frac{5}{18} t^{6}+\frac{2}{15} t^{5}-\frac{4}{45} t^{4}(t-1)^{5}
$$

and so

$$
\int_{0}^{1} \int_{0}^{1} G(t, s)^{2} d s d t=\frac{16}{14175} .
$$

Thus, in this special case, (3.8) takes the form

$$
L \frac{4 \sqrt{7}}{315}<1
$$

Condition (4.3) will be satisfied, for example, if $L \leq 29$. For an $f$ such as

$$
f(t, x):=20 \sin x+(t+1)^{2}
$$

the smallest constant $L$ that can be chosen so that $f$ satisfies $(3.4)$ on $[0,1] \times \mathbb{R}$ is $L=20$. The value $L=20$ does not satisfy Smirnov's condition (4.2), but it does satisfy (4.3).

Thus we can see that Theorem 7 and Theorem 8 apply to a wider class of problems than [7, Theorem 1].

Remark 7. If we let $k=0$ in (1.2) then we observe that we have classical two-point boundary conditions. In this case, the result of Theorem 7 leads to existence and uniqueness for the corresponding two-point problem with (3.5) becoming

$$
L\left(\int_{a}^{b}\left(\int_{a}^{b}|R(t, s)|^{q} d s\right)^{p / q} d t\right)^{1 / p}<1
$$


where $R$ is defined in (2.3). In this situation, with $p=2$ and $q=2$, the left hand side of (4.4) can be computed (by using Maple [1], for example) to obtain the equivalent condition

$$
L \frac{\sqrt{266}}{840}(b-a)^{3}<1
$$

This can then be compared with Smirnov's condition [7, Theorem 1] (with $k=0$ ), namely

$$
L(b-a)^{3} / 3<1,
$$

and with the condition (3.1) (with $k=0$ ), namely

$$
L \frac{2}{81}(b-a)^{3}<1
$$

Observe the restriction on the length of the interval and/or the Lipschitz constant in (4.6). Clearly, the inequality (4.5) is sharper than (4.6). Thus, for this special case, we can see that Theorem 7 applies to a wider class of problems than [7, Theorem 1]. Furthermore, we can see that our (4.5) is sharper than our (4.7).

Remark 8. Theorem 7 is sharper than Theorem 6 . However, as we have noted, the left-hand side of (3.5) may not be so straightforward to calculate in general situations. On the other hand, the left-hand side of (3.1) may be much easier to calculate. Thus, Theorem 6 still has advantages, despite its limitations when compared with Theorem 7.

Remark 9. Finally, we observe how our work not only confirms the importance of $L$ and $(b-a)$ as influencing factors in the existence and uniqueness of solutions to BVPs, but it also illustrates how the consideration of: the sign of Green's functions; estimates on Green's functions; and choice of metrics can play an important role.

\section{References}

[1] S.S. Almuthaybiri and C.C. Tisdell. Maple code for this paper. Available from Internet: https://drive.google.com/open?id= 1ZU9uK45vIvoYGZ1TBSgmXJUYWy7g5GHo.

[2] S.S. Almuthaybiri and C.C. Tisdell. Global existence theory for fractional differential equations: New advances via continuation methods for contractive maps. Analysis, 39(4):117-128, 2019. https://doi.org/10.1515/anly-2019-0027.

[3] S. Banach. Sur les opérations dans les ensembles abstraits et leur application aux équations intégrales. Fundamenta Mathematicae, 3:133-181, 1922. https://doi.org/10.4064/fm-3-1-133-181.

[4] O. Hölder. Über einen Mittelwertsatz. J Göttinger Nachr., pp. 38-47, 1889. (in German)

[5] L.J. Rogers. An extension of a certain theorem in inequalities. Messenger of Mathematics, 17:145-150, 1888. 
[6] I.A. Rus. On a fixed point theorem of Maia. Studia Univ. Babeş-Bolyai Math., 22:40-42, 1977.

[7] S. Smirnov. Green's function and existence of a unique solution for a third-order three-point boundary value problem. Math. Model. Anal., 24(2):171-178, 2019. https://doi.org/10.3846/mma.2019.012.

[8] C.C. Tisdell. Critical perspectives of pedagogical approaches to reversing the order of integration in double integrals. International Journal of Mathematical Education in Science and Technology, 48(8):1285-1292, 2017. https://doi.org/10.1080/0020739X.2017.1329559.

[9] C.C. Tisdell. On Picard's iteration method to solve differential equations and a pedagogical space for otherness. Internatational Journal Mathematical Education in Science and Technology, 50(5):788-799, 2019. https://doi.org/10.1080/0020739X.2018.1507051.

[10] E. Zeidler. Nonlinear functional analysis and its applications. I. Springer-Verlag, New York, 1986. 